\title{
Addendum to "Polynomial relations for cylindrical wheel stiffness characterization for use in a rolling noise prediction model"
}

\author{
Acta Acustica, 4, 4, https://doi.org/10.1051/aacus/2020003 \\ Matthew Edwards ${ }^{1, *}$, Fabien Chevillotte ${ }^{1}$, François-Xavier Bécot ${ }^{1}$, Luc Jaouen ${ }^{1}$, and Nicolas Totaro ${ }^{2}$ \\ ${ }^{1}$ Matelys Research Lab, 7 Rue des Maraîchers, Bât B, 69120 Vaulx-en-Velin, France \\ ${ }^{2}$ INSA-Lyon, 20 Avenue Albert Einstein, 69621 Villeurbanne cedex, France
}

Received 25 November 2020, Accepted 5 December 2020

The authors wish to correct three errors that appear in the above manuscript:

1. A typographical error was made in equation (8). The correct form is given below:

$$
f=r_{A} / r_{W} .
$$

$$
\begin{aligned}
P(f, g)=\frac{K}{w E} & =\exp \left(95.448 f^{5}+280.369 f^{4} g-1319.105 f^{3} g^{2}+4004.448 f^{2} g^{3}\right. \\
& -9965.202 f g^{4}+1052707.575 g^{5}-249.304 f^{4}-351.973 f^{3} g \\
& +1108.967 f^{2} g^{2}+75.910 f g^{3}-305716.234 g^{4}+246.535 f^{3} \\
& +123.059 f^{2} g-453.953 f g^{2}+33012.778 g^{3}-116.952 f^{2}+7.302 f g \\
& \left.-1625.255 g^{2}+28.714 f+42.606 g-4.643\right) .
\end{aligned}
$$

2. The rounded polynomial in equation (10) will not yield sufficiently precise results if evaluated as was originally presented. The equation with exact coefficients is given

\begin{tabular}{|c|c|c|c|c|c|c|}
\hline \multicolumn{7}{|c|}{$a / r_{W}[-]$} \\
\hline & 0.003 & 0.0037 & 0.0045 & 0.0056 & 0.0068 & 0.0084 \\
\hline \multicolumn{7}{|c|}{$\overline{r_{A} / r_{W}[-]}$} \\
\hline 0.15 & 0.113 & 0.115 & 0.12 & 0.124 & 0.127 & 0.136 \\
\hline 0.25 & 0.185 & 0.191 & 0.198 & 0.205 & 0.213 & 0.222 \\
\hline 0.35 & 0.244 & 0.254 & 0.263 & 0.274 & 0.284 & 0.296 \\
\hline 0.65 & 0.341 & 0.356 & 0.371 & 0.389 & 0.406 & 0.426 \\
\hline 0.75 & 0.365 & 0.382 & 0.399 & 0.418 & 0.441 & 0.463 \\
\hline 0.8 & 0.385 & 0.403 & 0.421 & 0.443 & 0.467 & 0.495 \\
\hline 0.825 & 0.395 & 0.416 & 0.435 & 0.46 & 0.484 & 0.517 \\
\hline 0.85 & 0.41 & 0.432 & 0.454 & 0.481 & 0.508 & 0.541 \\
\hline 0.87 & 0.426 & 0.448 & 0.472 & 0.502 & 0.531 & 0.567 \\
\hline 0.96 & 0.609 & 0.653 & 0.703 & 0.775 & 0.839 & 0.927 \\
\hline 0.97 & 0.685 & 0.74 & 0.806 & 0.889 & 0.974 & 1.086 \\
\hline 0.98 & 0.786 & 0.853 & 0.959 & 1.052 & 1.183 & 1.361 \\
\hline
\end{tabular}
below:
3. The exact values of the lookup table were not provided, making use of the lookup table method impossible as originally presented. They are given below:

Table 5. Lookup table exact values: $K_{\text {norm }}$.

*Corresponding author: matthew .edwards@matelys.com 
Table 5. (Continued)

\begin{tabular}{|c|c|c|c|c|c|c|}
\hline \multicolumn{7}{|c|}{$a / r_{W}[-]$} \\
\hline & 0.0103 & 0.0127 & 0.0156 & 0.0192 & 0.0236 & 0.029 \\
\hline \multicolumn{7}{|c|}{$\overline{r_{A} / r_{W}[-]}$} \\
\hline 0.15 & 0.14 & 0.144 & 0.152 & 0.158 & 0.165 & 0.171 \\
\hline 0.25 & 0.231 & 0.24 & 0.251 & 0.263 & 0.276 & 0.291 \\
\hline 0.35 & 0.309 & 0.323 & 0.338 & 0.355 & 0.374 & 0.395 \\
\hline 0.65 & 0.449 & 0.473 & 0.502 & 0.532 & 0.567 & 0.608 \\
\hline 0.75 & 0.492 & 0.522 & 0.554 & 0.596 & 0.638 & 0.689 \\
\hline 0.8 & 0.526 & 0.559 & 0.6 & 0.644 & 0.695 & 0.757 \\
\hline 0.825 & 0.548 & 0.586 & 0.629 & 0.679 & 0.737 & 0.804 \\
\hline 0.85 & 0.577 & 0.619 & 0.667 & 0.723 & 0.787 & 0.865 \\
\hline 0.87 & 0.607 & 0.654 & 0.707 & 0.768 & 0.842 & 0.93 \\
\hline 0.96 & 1.024 & 1.144 & 1.293 & 1.489 & 1.73 & 2.026 \\
\hline 0.97 & 1.192 & 1.364 & 1.572 & 1.846 & 2.183 & 2.598 \\
\hline 0.98 & 1.555 & 1.821 & 2.154 & 2.61 & 3.127 & 3.829 \\
\hline \multicolumn{7}{|c|}{$a / r_{W}[-]$} \\
\hline & 0.0357 & 0.0438 & 0.0539 & 0.0662 & 0.0814 & 0.1 \\
\hline \multicolumn{7}{|c|}{$r_{A} / r_{W}[-]$} \\
\hline 0.05 & 0.055 & 0.055 & 0.053 & 0.061 & 0.074 & 0.061 \\
\hline 0.15 & 0.179 & 0.19 & 0.201 & 0.213 & 0.227 & 0.24 \\
\hline 0.25 & 0.305 & 0.326 & 0.342 & 0.366 & 0.391 & 0.419 \\
\hline 0.35 & 0.418 & 0.444 & 0.474 & 0.506 & 0.542 & 0.586 \\
\hline 0.45 & 0.511 & 0.545 & 0.584 & 0.629 & 0.68 & 0.739 \\
\hline 0.89 & 1.143 & 1.292 & 1.478 & 1.708 & 2.014 & 2.407 \\
\hline 0.91 & 1.284 & 1.468 & 1.701 & 1.998 & 2.387 & 2.898 \\
\hline 0.93 & 1.527 & 1.771 & 2.09 & 2.499 & 3.036 & 3.749 \\
\hline 0.95 & 1.987 & 2.36 & 2.839 & 3.472 & 4.301 & 5.412 \\
\hline 0.96 & 2.406 & 2.892 & 3.54 & 4.376 & 5.491 & 6.954 \\
\hline 0.97 & 3.149 & 3.842 & 4.761 & 5.93 & 7.515 & 9.699 \\
\hline 0.98 & 4.726 & 5.838 & 7.325 & 9.28 & 11.923 & 15.805 \\
\hline
\end{tabular}

Cite this article as: Edwards M, Chevillotte F, Bécot F-X, Jaouen L \& Totaro N. 2021. Addendum to "Polynomial relations for cylindrical wheel stiffness characterization for use in a rolling noise prediction model". Acta Acustica, 5, 5. 